\title{
Análise espacial da Hanseníase na microrregião de Almenara, MG, Brasil*
}

\author{
Spacial analysis of Leprosy in the microregion of Almenara, MG, Brazil \\ Análisis espacial de la Lepra en la microregión de Almenara, MG, Brasil
}

Evaldo Pinheiro Amaral', Francisco Carlos Félix Lana'

'Universidade Federal de Minas Gerais. Departamento de Enfermagem. Belo Horizonte, MG

Submissão: 28/08/2008

Aprovação: 15/10/2008

\begin{abstract}
RESUMO
A hanseníase ainda é considerada um problema de saúde pública no Brasil. O objetivo deste estudo é analisar a situação epidemiológica da hanseníase na microrregião de Almenara/MG segundo sua distribuição espacial e suas relações com as condições socioeconômicas da população. Trata-se de um estudo ecológico. Os resultados colocam a microrregião como uma área hiperendêmica e indicam que os serviços de saúde não estão sendo capazes de detectar todos os casos existentes, contribuindo para manter a cadeia de transmissão da doença. A análise espacial identificou dois clusters, sendo um com coeficientes de detecção acima e outro abaixo do esperado. Acredita-se Que os resultados obtidos servirão para balizar a elaboração e implementação de medidas de prevenção e controle da hanseníase na região.
\end{abstract}

Descritores: Hanseníase/epidemiologia; Hanseníase/prevenção \& controle; Fatores socioeconômicos.

\section{ABSTRACT}

Hansen's disease is still considered a public health problem in Brazil. The objective of this ecological study is to analyze the epidemiological status of Hansen's disease in the microregion of Almenara, State of Minas Gerais, according to its spatial distribution and its relations with the socioeconomic conditions of the population. The results of this study classify the microregion as a hyperendemic area and indicate that the health services are not capable of detecting all existing cases, thus contributing to the maintenance of the disease transmission chain. The spatial analysis identified two clusters, one with detection coefficients above and the other below those expected. It is believed that the results of the study will help to establish guidelines for the development and implementation of prevention and control measures for Hansen's disease in the region.

Descriptors: Leprosy/epidemiology; Leprosy/prevention \& control; Socioeconomic factors.

\section{RESUMEN}

En Brasil, la hanseniasis todavía sigue siendo un problema de salud pública. El presente estudio ecológico tiene por objeto analizar la situación epidemiológica de la Hanseniasis en la microregión de Almenara, Estado de Minas Gerais, según su distribución espacial y su relación con las condiciones socioeconómicas de la población. Los resultados sitúan a la microregión en calidad de área híper endémica e indican Que como los servicios de salud no logran detectar todos los casos existentes persiste la cadena de transmisión de la enfermedad. El análisis espacial identificó dos clusters: uno con coeficientes de detección por arriba y otro por debajo de lo esperado. Los resultados obtenidos podrán servir de base para elaborar y poner en práctica medidas de prevención y control de la hanseniasis en la región.

Descriptores: Lepra/epidemiología; Lepra/prevención \& control; Factores socioeconómicos.

* Artigo extraído da dissertação de mestrado "Análise espacial da hanseníase na microrregião de Almenara, Minas Gerais: relações entre a situação epidemiológica e as condições socioeconômicas", apresentada à Escola de Enfermagem da UFMG em 2008. Pesquisa financiada com recursos do Conselho Nacional de Desenvolvimento Científico e Tecnológico/CNPQ, através do Edital MCT-CNPQ / MS-SCTIE-DECIT - N. ${ }^{\circ}$ 35/2005.

Correspondência: Francisco Carlos Félix Lana. Av. Alfredo Balena, 190. Bairro Santa Efigênia. CEP 30130-100. Belo Horizonte, MG. 


\section{INTRODUÇÃO}

A hanseníase é uma doença infecto-contagiosa, de evolução lenta, Que se manifesta principalmente através de sinais e sintomas dermatoneurológicos. Com a evolução da doença não tratada, surgem lesões nos nervos - principalmente os periféricos - Que acabam acarretando incapacidades físicas e deformidades. Estas, por sua vez, resultam não apenas em prejuízos econômicos e psicológicos aos doentes, mas também são responsáveis pelo preconceito Que recai sobre eles ${ }^{(1)}$.

Apesar de todos os esforços para sua eliminação, ela ainda é considerada um problema de saúde pública em alguns países em desenvolvimento, entre eles o Brasil ${ }^{(2)}$.

Em 2006, o Brasil apresentou coeficientes de prevalência e de detecção de 2,02 e 2, I I casos por 10.000 habitantes, respectivamente. Nesse mesmo ano, Minas Gerais contava com 2.278 casos de hanseníase em curso de tratamento, significando um coeficiente de prevalência de 1,2 casos em cada 10.000 habitantes $^{(3)}$. No entanto, estimou-se que 6.407 casos deixaram de ser diagnosticados e/ou registrados no período de 200 I a 2005, no Estado, o Que aumentaria sensivelmente esses valores ${ }^{(4)}$.

Entre as estratégias adotadas pela Secretaria de Estado da Saúde (SES/MG), está o estabelecimento de áreas consideradas prioritárias para o controle da endemia, definidas segundo critérios como a situação epidemiológica e a capacidade operacional. Entre os 33 municípios selecionados e acompanhados sistematicamente pelo Estado, 4 pertencem à microrregião de Almenara ${ }^{(3)}$. Essa microrregião faz parte do Vale do Jequitinhonha, considerada uma das regiões mais pobres do país. Seus municípios apresentam alguns dos piores indicadores socioeconômicos do Estado, como é o caso de Monte Formoso, cujo Índice de Desenvolvimento Humano (IDH) é igual a 0,570. Este valor é comparável aos de municípios do Norte e Nordeste do Brasil ${ }^{(5)}$.

Em 2006 foram notificados 115 novos casos da doença na microrregião, resultando em um coeficiente de detecção de 6,57 casos/10.000 habitantes, o Que a coloca como uma área hiperendêmica. Além disso, a distribuição da doença também não ocorre de forma homogênea na região $0^{(1,3)}$.

Sabe-se Que as condições sócio-econômicas e culturais têm grande influência na distribuição e propagação da endemia hansênica, apresentando uma estreita relação com as condições precárias de habitação, baixa escolaridade e ainda, com movimentos migratórios Que facilitam a difusão da doença. Nos países endêmicos, observamse diferenças na sua prevalência entre regiões, estados, microrregiões, municípios e, no caso de grandes cidades, entre espaços intra-urbanos, concentrando-se nos locais de maior pobreza. Deve-se ressaltar Que a avaliação da condição socioeconômica como fator de risco para a aQuisição de doenças é de grande importância por ser característica central na estrutura social das sociedades mais complexas, fornecendo informações essenciais para intervenções médicas e levantamentos epidemiológicos, além de sua importância no desenvolvimento de políticas públicas de saúde ${ }^{(6-8)}$.

Embora haja consenso sobre a relação entre as condições sociais e o desenvolvimento de doenças, é cada vez maior a necessidade de identificar indicadores específicos de condições de vida Que permitam associá-los. Desta forma é apropriado o estudo da associação e Quantificação entre indicadores pertencentes às esferas biológica e social no desenvolvimento de determinadas doenças como a hanseníase. Além disso, o estudo de sua distribuição espacial visando identificar grupos residentes em áreas Que ofereçam maior risco de adoecer pode auxiliar no planejamento, implementação, monitoramento e avaliação de ações voltadas para a prevenção e o controle dessa doençąa-10).

O geoprocessamento é uma importante ferramenta utilizada no planejamento de ações de prevenção e controle de doenças, particularmente da hanseníase. A Organização Pan-Americana de Saúde (OPAS) recomenda o uso deste recurso por países como Brasil e Índia, Que possuem grande extensão territorial e diferenças regionais acentuadas. Ela ainda reconhece o seu poder de análise para apoiar o planejamento, programação e avaliação de atividades e intervenções no setor saúde ${ }^{(11)}$. No Brasil, verifica-se um crescente interesse pela exploração de técnicas de representação espacial de dados na área de saúde e, em especial, da hanseníase ${ }^{(6-15)}$.

Dessa forma, o objetivo deste estudo foi analisar o comportamento espacial da hanseníase na microrregião de Almenara e a relação entre a situação epidemiológica da doença e os indicadores socioeconômicos da região.

\section{METODOLOGIA}

Trata-se de um estudo epidemiológico, do tipo ecológico, retrospectivo.

O cenário foi a microrregião de Almenara, situada no Vale do Jequitinhonha, região nordeste de Minas Gerais. Ela é composta por 16 municípios e, no ano 2000, possuía uma população total de aproximadamente de 175 mil habitantes ${ }^{(16)}$.

Foram analisados dados dos casos de hanseníase residentes nos municípios da microrregião de Almenara, notificados durante o período de 1998 a 2006. As informações foram coletadas diretamente das Fichas de Notificação de Hanseníase, disponibilizadas pela Coordenadoria de Dermatologia Sanitária (CEDS) da SES/MG. Esses dados foram lançados em um banco criado no software EPI-INFO (versão 6.02). A partir dele foram construídos os seguintes indicadores: coeficiente de detecção e proporção segundo grupo etário (< 15 anos, 15 anos), sexo, classificação operacional (paucibacilar, multibacilar), grau de incapacidade física e escolaridade ( $<4$ anos de estudo, 4 anos de estudo). A análise contemplou um estudo comparativo entre os indicadores encontrados e os parâmetros estabelecidos pelo Ministério da Saúde ${ }^{(1)}$ e também com resultados de outros trabalhos sobre o tema existentes na literatura científica.

Os dados socioeconômicos e as populações municipais do ano 2000 - desagregados por setores censitários - foram retirados das bases de dados do Censo 2000 da Fundação Instituto Brasileiro de Geografia e Estatística (IBGE) ${ }^{(16)}$.

Os dados sobre mortalidade dos anos de 1998, 1999 e $2000-$ desagregados por município - foram retirados do Sistema de Informação sobre Mortalidade (SIM).

A partir dos endereços informados nas Fichas de Notificação e tendo como base folhas e cartogramas fornecidos pelo IBGE, os casos de hanseníase foram georreferenciados aos respectivos setores censitários. Para isso, foram realizadas visitas à microrregião, etapa Que também contou com o auxílio de profissionais da área de saúde dos municípios estudados. 
As bases cartográficas em formato digital e georreferenciadas dos setores censitários rurais dos 16 municípios e dos setores urbanos do município de Almenara foram adeuiridas gratuitamente junto ao IBGE através de sua página na internet, na área de downloads. lá os setores urbanos dos demais 15 municípios, Que não estavam disponíveis na referida página, foram digitalizados por um profissional com formação na área de Geografia, tomando-se como base cartogramas e descrições dos limites dos setores.

Para se estimar a prevalência oculta da hanseníase na microrregião de Almenara utilizou-se a metodologia proposta por Suárez e Lombardi $^{(17)}$ e indicada pela OPAS/OMS ${ }^{(18)}$.

Para analisar a situação socioeconômica da população, elaborouse o Índice de Vulnerabilidade à Saúde (IVSA), adaptado a partir de metodologia aplicada pela Gerência de Epidemiologia e Informação da Prefeitura Municipal de Belo Horizonte ${ }^{(15)}$. Ele é composto por 13 indicadores agregados em níveis sucessivos e procura expressar o acesso da população a determinadas dimensões de cidadania e saúde. O seu desdobramento nos diversos níveis de agregação temática possibilita a identificação dos aspectos em Que a população de cada setor censitário está mais vulnerável ${ }^{(19)}$. Para o cálculo, cada variável foi escalonada entre os valores de 0 (zero) a I (um), em Que " 0 " indicava as menores vulnerabilidades e "I" as maiores. Em seguida, cada uma recebeu um peso relativo, chegando-se ao valor final do índice através da média ponderada dos escores. A partir dos valores do IVSA, os setores censitários da microrregião foram hierarquizados sendo, em seguida, classificados nas seguintes categorias: risco médio (com IVSA situado entre $\pm 1 / 2$ desvio-padrão em torno da média); risco baixo (com IVSA inferior aos do risco médio); risco elevado (com IVSA superior ao risco médio, até o limite de I desvio-padrão) e risco muito elevado (IVSA superior ao do risco elevado).

A associação dos endereços foi feita por setor censitário, sendo Que cada caso foi georreferenciado ao centróide de seu respectivo setor. Para se verificar a existência de conglomerados espaciais (clusters) a partir dos coeficientes médios de detecção da hanseníase, utilizou-se o índice I de Moran, método que mede a autocorrelação espacial de uma variável entre as áreas estudadas. O índice $I$ de Moran, na maioria dos casos, varia de $-1 \mathrm{a}+\mathrm{I}$, sendo Que, Quando não há correlação espacial, seu valor se aproxima de zero $^{(21)}$.

A identificação dos conglomerados foi feita através do método proposto por Kulldorff e Nagarwalla ${ }^{(22)}$, conhecido como estatística espacial de varredura. Para a aplicação desse método, foi realizada uma análise puramente espacial, utilizando-se o modelo de distribuição de Poisson. Para se obter um valor de "p" seguro, foram realizadas 999 interações. Um valor de p menor Que 0,001 foi considerado significante. Essas análises foram feitas através do SaTScan (versão 7.0.3).

Para se analisar a relação entre a distribuição espacial dos casos e as condições socioeconômicas, as médias dos coeficientes de detecção da hanseníase das Quatro categorias de risco (Baixo, Médio, Elevado, Muito Elevado) foram comparadas através do teste estatístico não-paramétrico de Kruskal-Wallis, visto Que estes dados não apresentavam distribuição normal. As diferenças entre elas foram consideradas estatisticamente significativas Quando o valor de " $p$ " foi menor que 0,05 .

Os mapas temáticos foram elaborados a partir do software MapInfo Professional (versão 7.5).
Este trabalho foi conduzido dentro dos padrões exigidos pela Declaração de Helsinque e da Resolução 196/1996, do Conselho Nacional de Saúde. O projeto foi submetido e aprovado pelo Comitê de Ética em Pesquisa da Universidade Federal de Minas Gerais (COEP-UFMG), por meio do parecer número ETIC 459/05.

\section{RESULTADOS}

Entre 1998 a 2006 foram notificados 889 casos de hanseníase na microrregião de Almenara, resultando numa detecção média no período de 5,7 casos por 10.000 habitantes. Este valor é superior às médias do Vale do Jequitinhonha, de Minas Gerais e do Brasil. A Tabela I traz os coeficientes de detecção de cada município, segundo o ano de notificação.

A média de idade entre os casos notificados foi de 40,6 anos ( \pm 19,8); 52,8\% $(n=469)$ eram do sexo feminino; $8,3 \%(n=74)$ eram em menores de 15 anos; $67,4 \%(n=598)$ eram multibacilares; $47,8 \%(n=424)$ dos casos avaliados $(n=888)$ apresentavam alguma incapacidade física no momento do diagnóstico $(9,7 \%$ no grau 2) e $52,7 \%(n=341)$ tinham menos de 4 anos de estudo completos, entre as fichas Que traziam essa informação $(n=647)$. Apenas 2,4\% $(n=21)$ dos doentes não foram notificados em seus municípios de residência.

Os homens apresentaram 1,48 vezes a prevalência de incapacidades físicas em relação às mulheres $(p<0,001)$. Entre os grupos etários, essa razão foi de 0,27 vezes dos menores de 15 anos em relação aos maiores ( $p<0,00 \mathrm{l}$ ). Quando se compara a escolaridade, os indivíduos com menos de 4 anos completos de estudo desenvolveram 1,32 vezes mais incapacidades físicas que aqueles com mais de 4 anos de estudo ( $p<0,001$ ).

Do total de fichas, $98,0 \%(n=871)$ traziam a informação do modo de descoberta dos casos novos. Destes, 89,8\% $(n=782)$ foram descobertos através de métodos de detecção passiva (encaminhamentos e demanda espontânea), contra apenas 10,2\% (n $=89$ ) nas formas ativas (exame de contatos e de coletividades). Os exames de contatos representaram apenas $8,1 \%(n=71)$.

Com base na metodologia utilizada, estimou-se que 289 casos de hanseníase deixaram de ser diagnosticados e/ou registrados entre os anos de 2002 a 2006.

Entre os 889 casos de hanseníase notificados de 1998 a 2006 de residentes na microrregião de Almenara, 96,3\% $(\mathrm{n}=866)$ puderam ser associados aos seus respectivos setores censitários. Os demais casos não puderam ser georreferenciados por problemas de inconsistência nos endereços informados nas Fichas de Notificação.

Ao todo, 126 setores censitários registraram pelo menos um caso de hanseníase durante o período de estudo, sendo 86 deles urbanos e 40 rurais. Os coeficientes médios de detecção variaram de 0,00 a 53,87 casos por 10.000 habitantes entre os setores e $88,1 \%(n=763)$ dos casos georreferenciados eram de residentes da zona urbana.

A Figura I mostra os coeficientes de detecção médios da hanseníase por setor censitário, mostrando Que a doença está concentrada, principalmente, nas porções central e leste da microrregião.

O valor médio encontrado para o IVSA foi de 0,426 , com desviopadrão de 0,138 . Os limites para cada uma das faixas de risco foram 
Tabela 1. Coeficientes de detecção da hanseníase por 10.000 habitantes na microrregião de Almenara, segundo município de residência e ano de notificação - 1998 a 2006.

\begin{tabular}{|c|c|c|c|c|c|c|c|c|c|c|}
\hline \multirow{2}{*}{ MUNICÍPIO } & \multicolumn{9}{|c|}{ ANO DE NOTIFICAÇÃO } & \multirow{2}{*}{ MÉDIA } \\
\hline & 1998 & 1999 & 2000 & 2001 & 2002 & 2003 & 2004 & 2005 & 2006 & \\
\hline Almenara & 14,4 & 16,9 & 10,5 & 10,4 & 12,0 & 17,5 & 13,0 & 15,6 & 12,6 & 13,6 \\
\hline Bandeira & 2,0 & 0,0 & 0,0 & 0,0 & 1,9 & 0,0 & 0,0 & 2,0 & 6,1 & 1,3 \\
\hline Divisópolis & 0,0 & 1,7 & 1,5 & 0,0 & 0,0 & 3,0 & 1,5 & 0,0 & 0,0 & 0,9 \\
\hline Felizburgo & 0,0 & 3,8 & 14,4 & 11,2 & 14,3 & 6,3 & 9,5 & 6,3 & 10,9 & 8,2 \\
\hline Jacinto & 3,4 & 6,0 & 5,0 & 1,66 & 5,8 & 1,7 & 1,7 & 2,5 & 11,6 & 4,4 \\
\hline Jequitinhonha & 2,1 & 1,2 & 1,3 & 0,0 & 0,4 & 0,4 & 0,4 & 0,9 & 0,4 & 0,8 \\
\hline Joaíma & 0,7 & 0,0 & 1,4 & 0,0 & 0,0 & 0,0 & 2,7 & 0,7 & 0,0 & 0,6 \\
\hline Jordânia & 2,9 & 5,8 & 4,1 & 1,00 & 3,0 & 6,0 & 8,0 & 12,9 & 8,9 & 5,9 \\
\hline Mata Verde & 0,0 & 0,0 & 2,8 & 0,0 & 0,0 & 1,3 & 0,0 & 0,0 & 2,5 & 0,9 \\
\hline Monte Formoso & 0,0 & 0,0 & 0,0 & 0,0 & 0,0 & 0,0 & 0,0 & 0,0 & 0,0 & 0,0 \\
\hline Palmópolis & 4,0 & 0,0 & 5,6 & 1,1 & 4,3 & 8,5 & 5,2 & 7,0 & 12,8 & 5,7 \\
\hline Rio do Prado & 0,0 & 0,0 & 3,7 & 0,0 & 13,4 & 0,0 & 0,0 & 0,0 & 2,1 & 2,1 \\
\hline Rubim & 9,5 & 10,8 & 6,2 & 8,4 & 3,3 & 22,5 & 11,9 & 17,8 & 9,0 & 11,0 \\
\hline Salto da Divisa & 1,4 & 10,1 & 1,5 & 9,0 & 1,5 & 4,6 & 4,7 & 1,6 & 9,9 & 4,9 \\
\hline Santa Maria do Salto & 27,0 & 4,2 & 14,7 & 26,5 & 9,1 & 27,4 & 10,9 & 18,1 & 23,5 & 18,0 \\
\hline Santo Antônio do Jacinto & 0,9 & 0,0 & 0,8 & 0,8 & 0,0 & 1,7 & 0,8 & 0,8 & 0,0 & 0,6 \\
\hline TOTAL & 5,2 & 5,5 & 5,0 & 4,5 & 4,9 & 7,4 & 5,5 & 6,6 & 7,0 & 5,7 \\
\hline
\end{tabular}

Fonte: Fichas de Notificação de Hanseníase - SES/MG

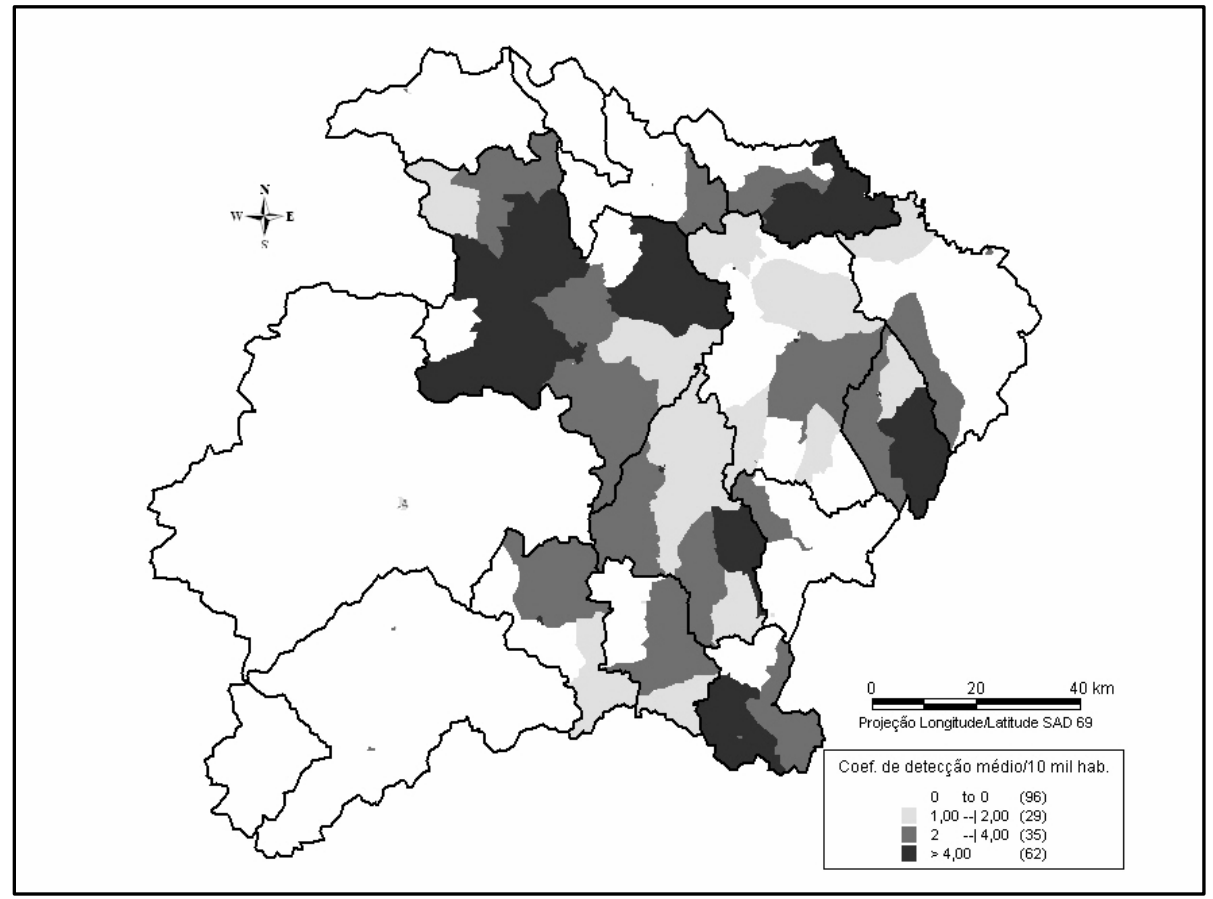

Figura 1. Mapa dos coeficientes de detecção médios da hanseníase dos setores censitários da microrregião de Almenara - 1998 a 2006.

os seguintes: Baixo Risco (0, 100 a 0,356); Médio Risco (0,357 a 0,496); Risco Elevado (0,497 a 0,633); Risco Muito Elevado (0,634 a 0,669$)$.

A maioria dos casos de hanseníase $(60,3 \%, n=522)$ estava localizada nos setores classificados como de baixo risco. Considerando-se também aQueles de risco médio, tem-se 90,2\% dos $\operatorname{casos}(\mathrm{n}=781)$.
A aplicação do teste de Kruskal-Wallis mostrou Que a média dos coeficientes de detecção da hanseníase nos setores pertencentes à categoria de Risco Baixo é maior que os das demais categorias e que a média da categoria de Risco Médio é maior que as de Risco Elevado e Muito Elevado $(p<0,05)$. Não houve diferenças estatisticamente significativas entre as médias dessas duas últimas categorias.

O valor calculado para o índice de Moran foi de 0,462 ( $<<0,001$ ), 


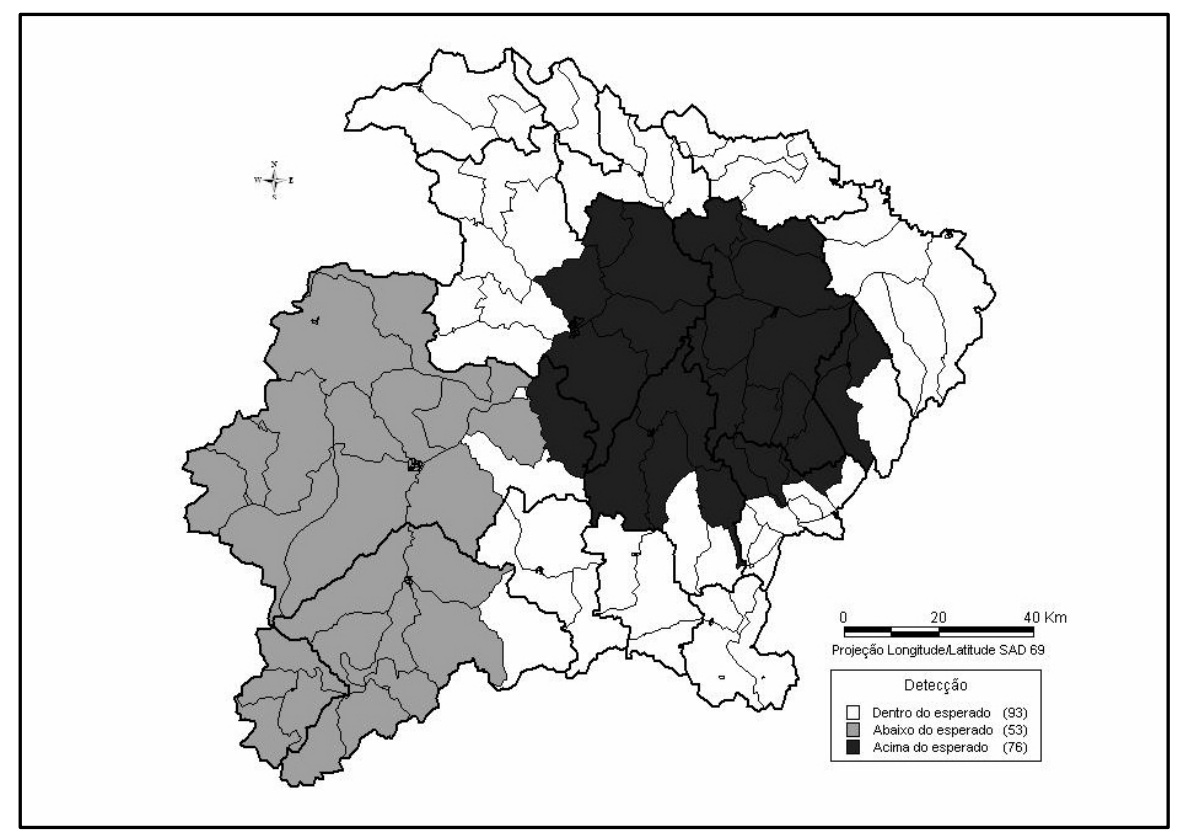

Figura 2. Mapa da distribuição dos setores censitários da microrregião de Almenarasegundo conglomerados de detecção da hanseníase.

mostrando a existência de autocorrelação espacial entre os setores censitários da microrregião de Almenara, em relação aos coeficientes médios de detecção da hanseníase. Através do teste de detecção de conglomerados, foi identificada a existência de dois clusters, sendo um deles com observação de casos da doença acima do esperado e outro cujo número de casos observados estava abaixo do esperado. A localização desses clusters está apresentada na Figura 2.

O conglomerado com coeficientes de detecção acima do esperado engloba o município de Jacinto e parte dos municípios de Almenara, Jequitinhonha, Rubim, Santo Antônio do Jacinto e Santa Maria do Salto. Já o conglomerado com coeficientes abaixo do esperado é formado por Monte Formoso e parte dos municípios de Joaíma e Jeeuitinhonha.

\section{DISCUSSÃO}

O coeficiente de detecção médio encontrado para a microrregião de Almenara permite classificá-la como uma região hiperendêmica ${ }^{(I)}$ e ratifica sua condição de área prioritária para o controle da endemia no Estado.

Ao se analisar os coeficientes de detecção mostrados na Tabela I, destacam-se Santa Maria do Salto, Almenara, Rubim e Felisburgo Que registraram coeficientes elevados em todos os anos e ocuparam os Quatro primeiro lugares, respectivamente, em relação à detecção. No caso de alguns municípios, como Rio do Prado e Salto da Divisa, observam-se grandes variações entre os municípios e no mesmo município, entre os diferentes anos estudados. Chama-se a atenção para o fato de Que, em municípios com menos de 20 mil habitantes, um pequeno número de casos novos acaba resultando em altos coeficientes de detecção, podendo mascarar a real situação epidemiológica $^{(23)}$. Apenas Monte Formoso não teve nenhum caso da doença notificado nesse período, condição Que chama a atenção já Que se trata de uma região endêmica. Kerr-Pontes et $\mathrm{al}^{(13)}$ observaram Que municípios com prevalências muito altas geralmente são rodeados por outros com prevalências também altas ou intermediárias.

A média de idade encontrada neste estudo é condizente com os dados existentes na literatura que mostram que a hanseníase acomete principalmente indivíduos na faixa etária de 25 a 54 anos $^{(24,25)}$. Apesar de ser considerada uma doença do adulto e do adulto jovem, muitos casos são registrados em crianças e jovens, situação Que pode indicar um aumento na cadeia de transmissão do bacilo e/ou uma deficiência nos serviços de vigilância e controle da doença ${ }^{(26)}$.

O percentual de casos em menores de Quinze anos chama a atenção, pois Pereira |r. e Torrecila ${ }^{(27)}$ indicaram a realização de exames em escolares Quando mais de $8 \%$ do total de casos forem detectados em menores de 15 anos. O resultado encontrado justifica a adoção de medidas de prevenção e controle mais específicas voltadas para esse grupo da população, como a busca ativa em escolas e creches, a realização de palestras esclarecendo sinais e sintomas da doença e a intensificação dos exames de comunicantes. Enfatiza-se a necessidade de ineuéritos populacionais cuidadosos nestas idades, tanto para a detecção de casos como para seu melhor seguimento em áreas endêmicas ${ }^{(28)}$. Além disso, um controle rigoroso envolvendo a vigilância epidemiológica deve ser mantido em crianças sob risco de contrair a hanseníase, no sentido de detectar a doença mais precocemente $^{(26)}$.

Houve predomínio das formas clínicas multibacilares em relação às paucibacilares. Este resultado poderia ser visto, dentro de um enfoque epidemiológico, como um indicativo de estabilização da endemia ou tendendo a uma situação de baixa prevalência ${ }^{(29)}$. Por outro lado, isso poderia ser um sinal de Que o diagnóstico tem ocorrido tardiamente, colaborando para a manutenção da cadeia de transmissão da doença, já Que estas são consideradas as principais 
fontes de infecção da hanseníase.

As incapacidades físicas e deformidades são os principais problemas da hanseníase. Considerando Que essa doença atinge em maior proporção as pessoas em idade produtiva, o desenvolvimento de incapacidades pode interferir drasticamente em suas atividades laborais, sob risco de excluí-las da cadeia produtiva, resultando em perdas não apenas econômicas, mas também sociais e psicológicas, impactando negativamente na Qualidade de vida ${ }^{(25,30)}$. Por essa razão, deve-se dar atenção especial aos casos diagnosticados já com alguma incapacidade física, inclusive no grau I, visto Que estas já podem trazer limitações à vida dos indivíduos, bem como evoluir para formas mais graves, se não forem corretamente tratadas.

A maior ocorrência de incapacidades no sexo masculino pode estar relacionada ao menor acesso aos serviços de saúde já Que estes oferecem mais programas voltados para a saúde da mulher, bem como a menor preocupação dos homens em relação ao corpo e à estética, o Que propiciaria demora no diagnóstico ${ }^{(31)}$.

Embora os doentes menores de 15 anos tenham sido menos acometidos por incapacidades físicas Que os maiores, ainda assim o número encontrado é preocupante, visto Que as repercussões negativas da hanseníase na vida do indivíduo podem se tornar mais graves ao acometê-lo ainda na infância ${ }^{(32)}$.

Os resultados encontrados neste estudo confirmam a baixa escolaridade como um fator de risco para desenvolver as formas incapacitantes da hanseníase. Esta variável deve ser levada em consideração pelos serviços de saúde no planejamento e desenvolvimento de suas atividades, principalmente na elaboração de ações educativas Que visem ensinar sobre a doença e as formas de prevenção e tratamento das incapacidades. Considerando tratarse de um grupo com baixa escolaridade, a abordagem a esses pacientes deve se adequar às suas capacidades cognitivas e aos seus conhecimentos e crenças $^{(4,30,33)}$.

O modo de descoberta dos casos novos é um indicador operacional relacionado à forma como os serviços de saúde se organizam para prestar a assistência de saúde em hanseníase. Pinto Neto e Villa ${ }^{(33)}$ viram aspectos positivos no comparecimento voluntário dos doentes às unidades de saúde, uma vez Que isso refletiria uma população bem informada em relação à doença. lá para Lana et $\mathrm{al}^{(31)}$ a passividade dos métodos de detecção pode ser erroneamente interpretada como diminuição do coeficiente de detecção, contribuindo para aumentar o número de casos não diagnosticados. Pinto Neto e Villa ${ }^{(33)}$ afirmaram Que, apesar da importância dos comunicantes na cadeia epidemiológica da hanseníase, as atividades relacionadas ao controle dos mesmos têm sido pouco valorizadas pelos serviços e profissionais de saúde.

A estimativa dos casos não detectados representaria um acréscimo de 52,9\% na prevalência registrada e, ainda, significaria dizer Que $34,6 \%$ dos doentes permaneceram sem diagnóstico durante o período, podendo atuar como fontes de contágio e dando continuidade à cadeia de transmissão da doença na região.

A distribuição dos casos pelos setores censitários mostra Que esta não ocorre de forma homogênea pela microrregião, além de confirmar a natureza urbana da endemia.

A concentração dos casos nos setores classificados como de Baixo e Médio Risco pode parecer paradoxal, visto Que essas áreas, teoricamente, seriam as menos vulneráveis aos problemas de saúde. No entanto, uma possível explicação para essa situação seria a de Que as regiões de menor vulnerabilidade geralmente apresentam os melhores indicadores socioeconômicos e tendem a ter serviços de saúde mais organizados. Conseqüentemente, seriam capazes de detectar os casos da doença de forma mais eficiente ${ }^{(7)}$.

Outra hipótese para esse resultado seria de Que, apesar de existirem peQuenas diferenças entre os municípios, no geral, todos estão inseridos em uma região carente e apresentam baixos Índices de Desenvolvimento Humano. Dessa forma, a peQuena diferença existente entre suas condições de vulnerabilidade - relativizada através da criação do IVSA - poderia gerar um viés na análise dos dados.

A existência de um conglomerado (cluster) com coeficientes de detecção médios acima do esperado poderia indica uma maior força de transmissão da hanseníase nessa área. Ela também sugeriria Que os serviços de saúde locais foram mais eficientes em diagnosticar os casos ali existentes. Por outro lado, o conglomerado com coeficientes de detecção médios abaixo do esperado poderia evidenciar a ocorrência de falhas nos serviços Que acabam resultando em subnotificação e/ou diagnóstico tardio. Ele serviria de alerta para a necessidade de se intensificar as ações de busca ativa nesses municípios, a fim de detectar um número maior de casos e de forma mais precoce.

\section{CONSIDERAÇÕES FINAIS}

Entre as limitações deste estudo cabe destacar a utilização de dados secundários Que muitas vezes apresentam inconsistência em relação à Quantidade e Qualidade de suas informações. Apesar disso, a escolha por esse tipo de fonte reduz os custos operacionais e não inviabiliza a realização de análises. Outro obstáculo encontrado foi a não disponibilidade das bases cartográficas digitalizadas e georreferenciadas das zonas urbanas dos municípios com populações inferiores a 25 mil habitantes, o Que demandou a contratação de um profissional da área de cartografia para a elaboração das mesmas.

A partir dos resultados encontrados, conclui-se que a estrutura e organização dos serviços de saúde têm maior influência no desenho da atual situação epidemiológica da hanseníase na microrregião de Almenara do Que os fatores socioeconômicos. Dessa forma, o desafio Que se apresenta é o de reestruturar o modelo de assistência à saúde, de forma a torná-lo mais ágil e resolutivo, garantindo o acesso facilitado aos portadores da doença. Essa reestruturação deve ser fundamentada por políticas públicas efetivas Que garantam a sustentabilidade econômica, política e social do Programa de Controle, bem como o apoio financeiro a pesquisas nessa área.

\section{REFERÊNCIAS}

1. Ministério da Saúde (BR). Secretaria de Políticas de Saúde. Departamento de Atenção Básica. Guia para o controle da hanseníase. Brasília: Ministério da Saúde; 2002.

2. World Health Organization. Global strategy for further reducing the leprosy burden and sustaining leprosy control activities: plan period 2006-2010. Geneva: WHO; 2005.

3. Secretaria de Estado da Saúde de Minas Gerais. Coordenadoria Estadual de Dermatologia Sanitária. Encontro Estadual 2007 - 
Hanseníase: procurar para curar. Belo Horizonte: Secretaria de Estado da Saúde; 2007.

4. Moschioni C. Fatores de risco para incapacidade física anotados no momento do diagnóstico de 19.823 casos de hanseníase, no período de 2000 a 2005, em Minas Gerais, Brasil [dissertação]. Belo Horizonte (MG): Faculdade de Medicina, Universidade Federal de Minas Gerais; 2007.

5. Martins RB, Libânio IC. Atlas do Desenvolvimento Humano no Brasil [CD-ROM]. Belo Horizonte: ESM Consultoria; 2003.

6. Lapa T, Ximenes R, Silva NN, Souza W, Albuquerque MFM, Campozana G. Vigilância da hanseníase em Olinda, Brasil, utilizando técnicas de análise espacial. Cad Saúde Pública 200 I; 17(5): 1153-62.

7. Montenegro ACD, Werneck GL, Kerr-Pontes LRS, Barreto ML, Feldmeier $\mathrm{H}$. Spatial analysis of the distribution of leprosy in the State of Ceará, Northeast Brazil. Mem Inst Oswaldo Cruz 2004; 99(7): 683-6.

8. Dias MCFS, Nobre ML, Dias GH. Distribuição espacial da hanseníase no município de Mossoró/RN, utilizando o Sistema de Informação Geográfica - SIG. An Bras Dermatol 2005; 80(Supl. 3): S289-94.

9. Souza WV, Barcellos CC, Brito AM, Carvalho MS, Cruz OG, Albuquerque MFM, et al. Aplicação do modelo bayesiano empírico na análise espacial da ocorrência da hanseníase. Rev Saúde Pública 200 I; 35(5): 474-80.

10. Opromolla PA, Dalben I, Cardim M. Análise da distribuição espacial da hanseníase no Estado de São Paulo, 1991-2002. Rev Bras Epidemiol 2005; 8(4): 356-64.

11. Organización Panamericana de la Salud. Uso de los Sistemas de Información Geográfica en Epidemiologia (SIG-EPI). Boletín Epidemiol 1996; 17(1): 1-6.

12. Mencaroni DA. Análise espacial da endemia hansênica no município de Fernandópolis/SP [dissertação]. Ribeirão Preto (SP): Escola de Enfermagem, Universidade de São Paulo; 2003.

13. Kerr-Pontes LRS, Montenegro ACD, Barreto ML, Werneck GL, Feldmeier $\mathrm{H}$. Inequality and leprosy in Northeast Brazil: an ecological study. Int I Epidemiol 2004; 33(2): 262-9.

14. Santos CB, Hino P, Cunha TN, Villa TCS, Muniz JN. Utilização de um Sistema de Informação Geográfica para descrição dos casos de tuberculose. Bol Pneumol Sanit 2004; 12(1): 5-10.

15. Gauy IS, Hino P, Santos CB. Spatial distribution of leprosy cases in Ribeirão Preto, Brazil, 2004. Rev Latino-Am Enfermagem 2007; 15(3): 460-5.

16. Fundação Instituto Brasileiro de Geografia e Estatística. Censo Demográfico 2000 - Agregado por setores censitários dos resultados do universo. Rio de Janeiro: IBGE; 2003.

17. Suárez REG, Lombardi C. Estimado de prevalencia de lepra. Hansen Int 1997; 22(2): 31 -4.

18. Organização Pan-Americana de Saúde. Hanseníase hoje: Boletim de Eliminação na Hanseníase nas Américas. Brasília: OPAS; 1998.

19. Prefeitura Municipal de Belo Horizonte. Gerência de Epidemiologia e Informação. Índice de Vulnerabilidade à Saúde. Belo Horizonte (MG): PBH; 2003.

20. Prefeitura Municipal de Belo Horizonte. Gerência de Planejamento e Desenvolvimento. Plano Municipal de Saúde de Belo Horizonte: 2005-2008. Belo Horizonte: PBH; 2005.

21. Cliff AD, Ord IK. Spatial processes: models and applications. I. ed. London: Pion; 1981.

22. Kulldorff M, Nagarwalla N. Spatial disease clusters: detection and inference. Stat Med 1995; 14(8): 799-810.

23. Lastória IC, Putinatti MSMA. Utilização de busca ativa de hanseníase: relato de uma experiência de abordagem na detecção de casos novos. Hansen Int 2004; 29(1): 6- I I.

24. Hinrichsen SL, Pinheiro MRS, Jucá MB, Rolim H, Danda GIN, Danda DMR. Aspectos epidemiológicos da hanseníase na cidade de Recife, PE em 2002. An Bras Dermatol 2004; 79(4): 4I 3-2 I.

25. Gomes CCD, Pontes MAA, Gonçalves HS, Penna GO. Perfil clínico-epidemiológico dos pacientes diagnosticados com hanseníase em um centro de referência na região nordeste do Brasil. An Bras Dermatol 2005; 80(Supl. 3): S283-8.

26. Ferreira IN, Alvarez RRA. Hanseníase em menores de 15 anos no município de Paracatu, MG (1994 a 2001). Rev Bras Epidemiol 2005; 8(1): 41-9.

27. Pereira Jr AC, Torrecila MA. Profilaxia da hanseníase. In: Talhari N, Neves RG, organizadores. Dermatologia Tropical: Hanseníase. 3a. ed. Manaus: Gráfica Tropical; 1997. p. 15 I-2.

28. Selvasekar A, Geetha J, Nisha K, Manimozhi N, Jesudasan K, Rao PS. Childhood leprosy in endemic área. Lepr Rev 1999; 56(3): 600- 14.

29. Oliveira MLW, Motta CP. A hanseníase como problema de saúde pública. In: Lombardi $\mathrm{C}$, organizador. Hanseníase: epidemiologia e controle. Ia ed. São Paulo: Imprensa Oficial do Estado; 1990. p. 13-32.

30. Duarte MTC, Ayres IA, Simonetti JP. Socioeconomic and demographic profile of leprosy carriers attended in nursing consultations. Rev Latino-Am Enfermagem 2007; 15 (esp): 774-9.

31. Lana FCF, Velásquez-Melendéz JG, Lanza FM, Branco AC, Teixeira S, Malaquias LC et al. Transmissão e controle da hanseníase no município de Governador Valadares/MG - Período de 1990 a 2000. Hansen Int 2002; 27(2): 83-92.

32. Lana FCF, Amaral EP, Lanza FM, Lima PL, Carvalho ACN, Diniz LG. Hanseníase em menores de 15 anos no Vale do JeQuitinhonha, Minas Gerais Brasil. Rev Bras Enferm 2007; 60(6): 696-700.

33. Pinto Neto IM, Villa TCS. Características epidemiológicas dos comunicantes de hanseníase Que desenvolveram a doença, notificados no Centro de Saúde de Fernandópolis (1993 a 1997). Hansen Int 1999; 24(2): 129-36. 